\title{
Sector Imaging Radar for Enhanced Vision
}

\author{
G. Krieger, J. Mittermayer, A. Moreira, M. Wendler, F. Witte, W. Keydel \\ Institut für Hochfrequenztechnik und Radarsysteme \\ Deutsches Zentrum für Luft- und Raumfahrt (DLR) e.V. \\ P.O. Box 1116, D-82230 Wessling, Germany \\ E-mail: Gerhard.Krieger@dlr.de
}

\begin{abstract}
SIREV (Sector Imaging Radar for Enhanced Vision) is an innovative radar system which can supply high-quality images of a sector in front of an aircraft under almost every weather condition. In contrast to synthetic aperture radar $(S A R)$ systems, no relative motion between sensor and targets is required in SIREV. The high frame repetition frequency in SIREV allows to detect even very rapid changes in the imaged scenes. Besides a map of the earth's surface, the complex-valued radar images can be further processed to supply additional information about the topography and objects in the field of view. A fast processing algorithm has been developed for SIREV which allows a very accurate, phase preserving and efficient image formation. Raw data were obtained by a first demonstration flight using a helicopter as a platform and the data processing results show good agreement with the theory. Motion errors of the platform could be extracted from the range compressed raw data avoiding the need of an inertial navigation system. After the image formation, coherent and incoherent image averaging processes have been applied to improve the image quality. The evaluation of the computational effort shows that a real-time hardware realization can be carried out using off-the-shelf digital components.
\end{abstract}

\section{Introduction}

Adverse weather conditions affect both flight safety and the operational area of airborne platforms. This problem becomes most evident during take-off, landing and taxiing, but also reliable collision warning in airspace gains increasing importance. In all these cases it would be highly desirable to provide the pilot with visual information about the surrounding environment independent of the weather conditions. A weather independent imaging system will also substantially increase the situation awareness of the crew without the necessity to recourse to an external electronic guidance system. Available radar systems, like the synthetic aperture radar (SAR), have proved as a valuable tool in many applications where weather independent imaging is required, but, inherent to the underlying principle, these systems suffer from a visualization gap with respect to the forward looking direction [2][9]. In order to fill this blind spot a new radar system called Sector Imaging Radar for Enhanced Vision (SIREV) has been developed at the German Aerospace Center [1][10]. This innovative system has the potential to supply high quality radar images of a sector in front of the aircraft as shown in Figure 1.

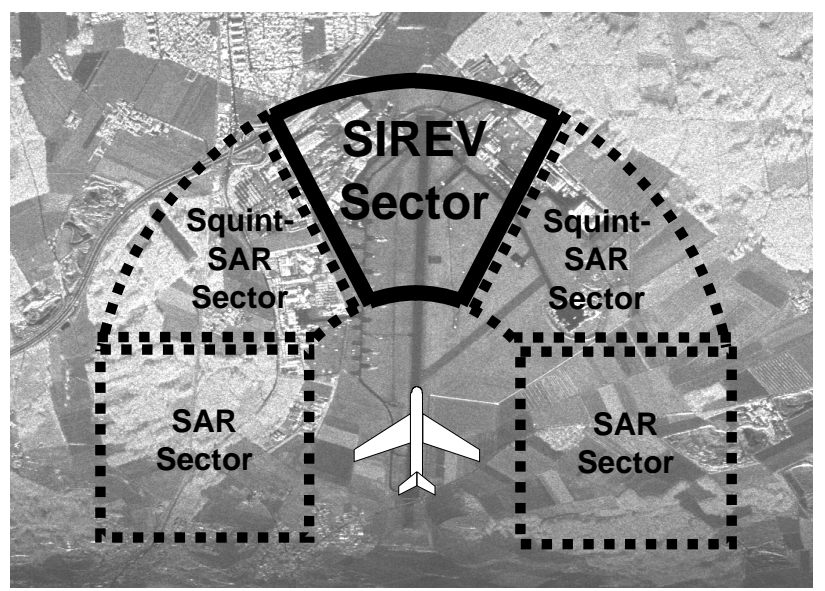

Figure 1: SIREV is an innovative radar system which has the capability to provide high quality images of a sector in front of an aircraft independent of the weather conditions. Since SAR or Squint SAR systems have no azimuth resolution in flight direction their operational area is restricted to applications where boresight vision is sufficient.

SIREV originated from a time to space mapping of the SAR principle by replacing the virtual antennas in SAR with a set of physically existent antenna elements distributed in space [11]. This array may be arranged and operated in many different ways. Most akin to a SAR system is the monostatic mode where each element has both transmitting and receiving capabilities. By switching 
the elements sequentially, image formation may be achieved by a coherent processing of the radar echoes similar to a conventional SAR system [2]. Alternatively, the radar echoes may be recorded simultaneously by all array elements which will increase the performance of the system. The disadvantage of these operational modes is that a $T / R$ module is required for each array element which increases the complexity and costs of the necessary hardware. A more cost-effective realization may be achieved by using only one transmit antenna, which leads to a bistatic configuration where the receive antennas are spatially separated from the transmit antenna. This configuration can also be operated in either a simultaneous or a sequential mode. The simultaneous recording makes optimum use of the transmitted signal power by a large effective antenna area, whereas the sequential mode minimizes the hardware requirements. The major drawback of the bistatic configuration with one transmit antenna is, that it reaches only half of the image resolution as compared to the monostatic case. In principle, it is also possible to combine the advantages of the monostatic and bistatic configurations using a small number of transmit antennas.

\section{SIREV geometry and data acquisition}

The current SIREV system consists of one transmit antenna and a linear array of receiving patch elements as shown in Figure 2. The receive antennas are centered around the across-track position $y=0$ at height $z=h$, while the transmit antenna is at position $x=0, y=0$ and $z=h-z_{\text {offset }}$. The direction parallel to the receiving array is termed azimuth and the direction orthogonal to azimuth in forward direction, slantwise to the ground, is denoted as slant range. Correspondingly, the direction of the $x$-axis is termed ground range.

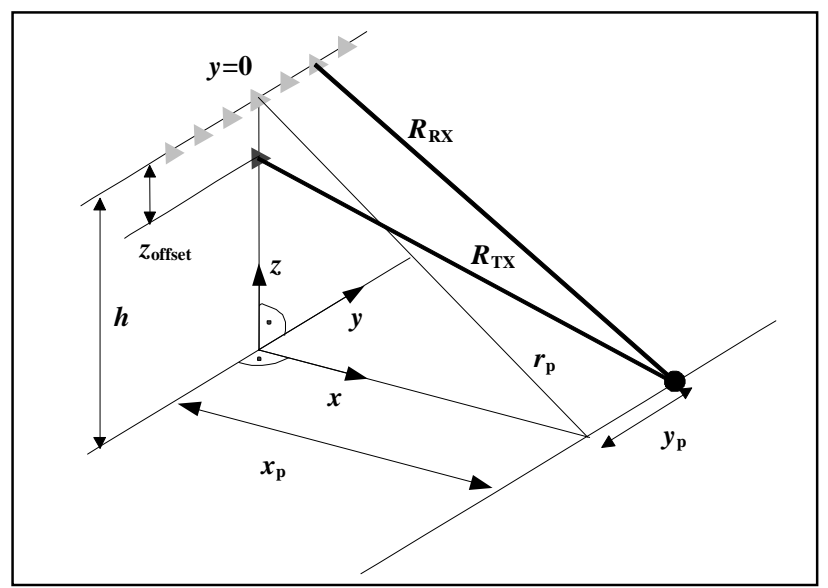

For a point target at ground range position $x_{p}$ and azimuth position $y_{p}$ the transmit and receive paths $R_{T X}$ and $R_{R X}$ of the radar signal are given by

$$
\begin{gathered}
R_{\mathrm{TX}}=\sqrt{x_{p}{ }^{2}+y_{p}{ }^{2}+\left(h-z_{\text {offset }}\right)^{2}}=\sqrt{r_{p}{ }^{2}+y_{p}{ }^{2}+z_{\text {offset }}^{2}-2 \cdot h \cdot z_{\text {offset }}} \\
R_{\mathrm{RX}}=\sqrt{x_{p}{ }^{2}+\left(y_{p}-y_{i}\right)^{2}+h^{2}}=\sqrt{r_{p}{ }^{2}+\left(y_{p}-y_{i}\right)^{2}}
\end{gathered}
$$

where for convenience the point target slant range position $r_{p}=\sqrt{x_{p}^{2}+h^{2}}$ has been introduced. The variables $y_{i}$ denote the positions of the receiving antenna elements.

Using the definitions for the signal transmit and receive paths, the SIREV signal modulation of a point target at position $r_{p}$ and $y_{p}$ can be written as

$$
s\left(y_{i}, \tau ; y_{p}, r_{p}\right)=C \cdot \exp \left[j \pi k_{e}\left(\tau-\frac{R_{T X}+R_{R X}}{c_{o}}\right)^{2}\right] \cdot \exp \left[-j \frac{2 \pi}{\lambda}\left(R_{T X}+R_{R X}\right)\right]
$$

where $\tau$ denotes the range time, $c_{0}$ is the velocity of light, and $\lambda$ is the wavelength. The first exponential term describes the linear frequency modulation of a range chirp with modulation rate $k_{e}$. The azimuth modulation is given by the second exponential term. The contributions from the pulse envelope and the antenna weighting have been omitted in this equation since the phase functions are more important for the development of the processing algorithms.

\section{SIREV processing}

\subsection{Spatial domain focusing}

In general, the focusing of the SIREV raw data may be achieved by a space-variant 2-D correlation

$$
u(y, r)=\sum_{i=1}^{N_{a}} \int_{0}^{+\infty} h\left(y_{i}, \tau ; y, r\right) \cdot s\left(y_{i}, \tau\right) \cdot d \tau
$$

where $s\left(y_{i}, \tau\right)$ is the unfocused raw data signal recorded by the $N_{a}$ antenna elements and $u(y, r)$ is the processed complex image expressed in terms of azimuth $y$ and slant range $r$. According to the matched filter concept, the space-variant 2-D kernel $h\left(y_{i}, \tau ; y, r\right)$ is given by the complex conjugate of the predicted response of an ideal point scatterer located at $(y, r)$, i.e., $h\left(y_{i}, \tau ; y, r\right)=s^{*}\left(y_{i}, \tau ; y, r\right)$. While such a 2-D focusing approach maximizes the signal-to-noise ratio under the reasonable assumption of second-order white background noise, it is also most expensive from a computational point of view, since a two-dimensional integration has to be performed for each pixel in the final image.

Figure 2: Basic SIREV geometry 
A computationally more efficient focusing algorithm may be derived, if the received raw data signals are first compressed in range by a 1-D convolution:

$$
u_{r c}\left(y_{i}, r\right)=\int_{0}^{T_{p}} h_{r}(\tau) \cdot s\left(y_{i}, \frac{r}{c_{0}}-\tau\right) \cdot d \tau
$$

Here, $h_{r}(\tau)$ is the time-reversed complex conjugate of the transmitted chirp of duration $T_{p}$. The range compressed signal is denoted by $u_{r c}\left(y_{i}, r\right)$. Since the convolution in this equation is time-invariant, it may efficiently be implemented in the frequency domain by taking advantage of the Fast Fourier Transform (FFT). After range compression, the response of a point scatterer located at $\left(y_{p}, r_{p}\right)$ may well be approximated by a set of $\delta$-impulses at $r\left(y_{i}, y_{p}, r_{p}\right)=R_{T X}\left(y_{p}, r_{p}\right)+R_{R X}\left(y_{i} ; y_{p}, r_{p}\right)$. Thus, azimuth compression is achieved by the 1-D space variant correlation:

$$
u(y, r)=\sum_{i=1}^{N_{a}} h_{a}\left(y_{i} ; y, r\right) \cdot u_{r c}\left[y_{i}, r\left(y_{i} ; y, r\right)\right]
$$

where the kernel $h_{a}$ is given by

$$
h_{a}\left(y_{i} ; y, r\right)=\exp \left[j \frac{2 \pi}{\lambda}\left(\sqrt{r^{2}+y^{2}+z_{\text {offset }}^{2}-2 h z_{\text {offset }}}+\sqrt{r^{2}+\left(y_{i}-y\right)^{2}}\right)\right]
$$

In this approach, the inseparable and shift-variant 2-D correlation has been approximated by two 1-D correlations, resulting in a considerably reduced computational effort. For a large number of antenna elements, the efficiency may be further increased by an appropriate adaptation of the Extended Chirp Scaling Algorithm [8][6], which is introduced in the next section.

\subsection{Extended chirp scaling algorithm for SIREV}

An efficient processing algorithm for SIREV has been derived from a reformulation of the Extended Chirp Scaling algorithm [7][8][6]. Figure 3 summarizes the basic processing steps. As can be seen from the block diagram, the algorithm basically consists of Fourier transforms and complex multiplications, thus allowing an efficient implementation which takes advantage of the FFT algorithm. It is also possible to integrate a motion compensation into the processing as shown by the gray boxes. A detailed description of the algorithm and a derivation of the associated scaling functions may be found in [7].

\section{Estimation of image quality}

In order to obtain a reference for the SIREV image data processing and to get a first impression of the SIREV mapping capabilities a SIREV image simulation has been carried out based on X-Band data from the Experimental SAR system of DLR (E-SAR) [5].

The results of this simulation are shown in Table 1 (cf. [7] and [1]). As a main parameter, the wavelength decreases from left to right. From the simulations it becomes apparent that the image quality will substantially improve for higher radar frequencies if the antenna length is kept constant.

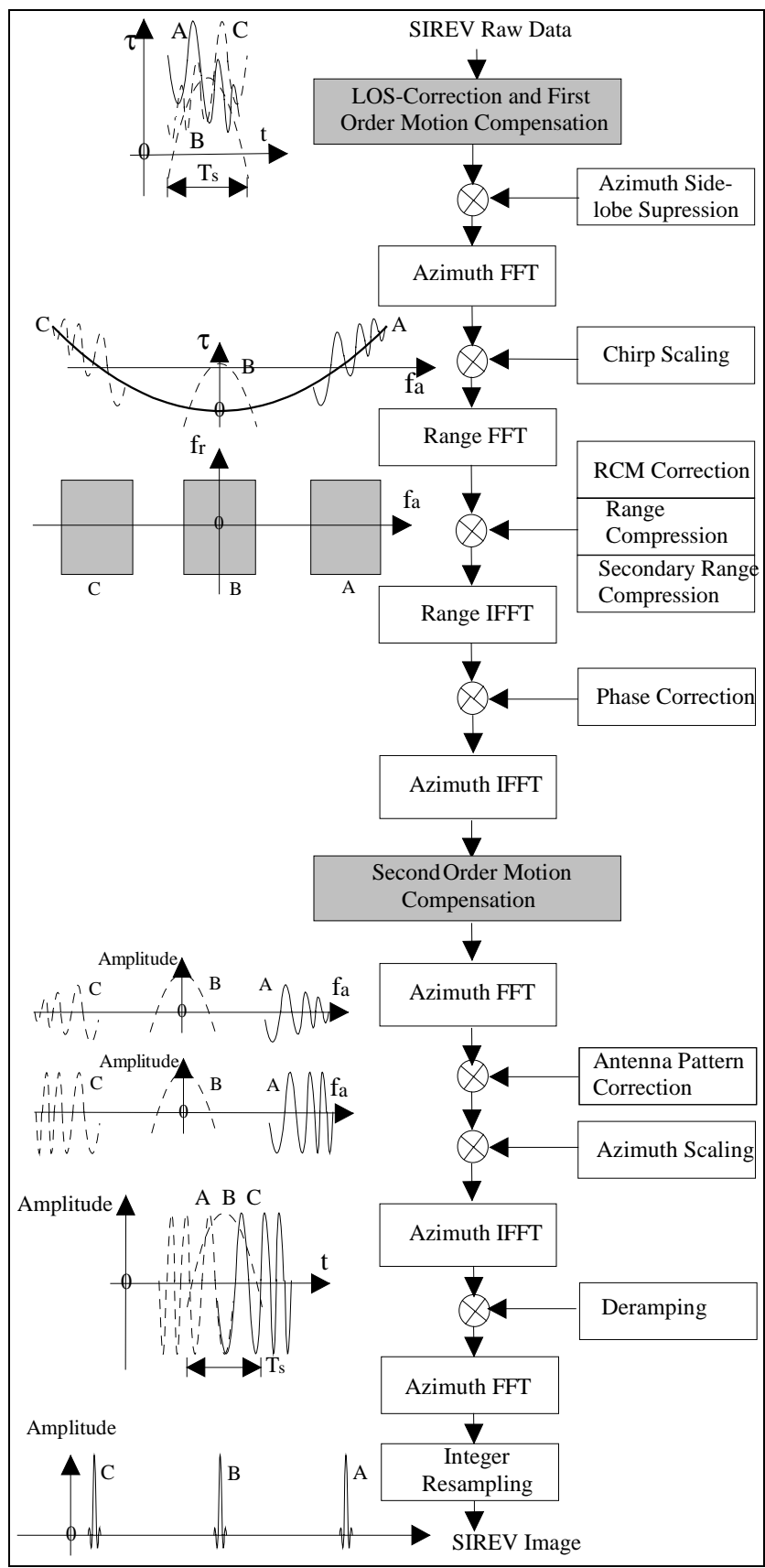

Figure 3: Ekended Chirp Scaling algorithm for SIREV (for a detailed description see [7]). 


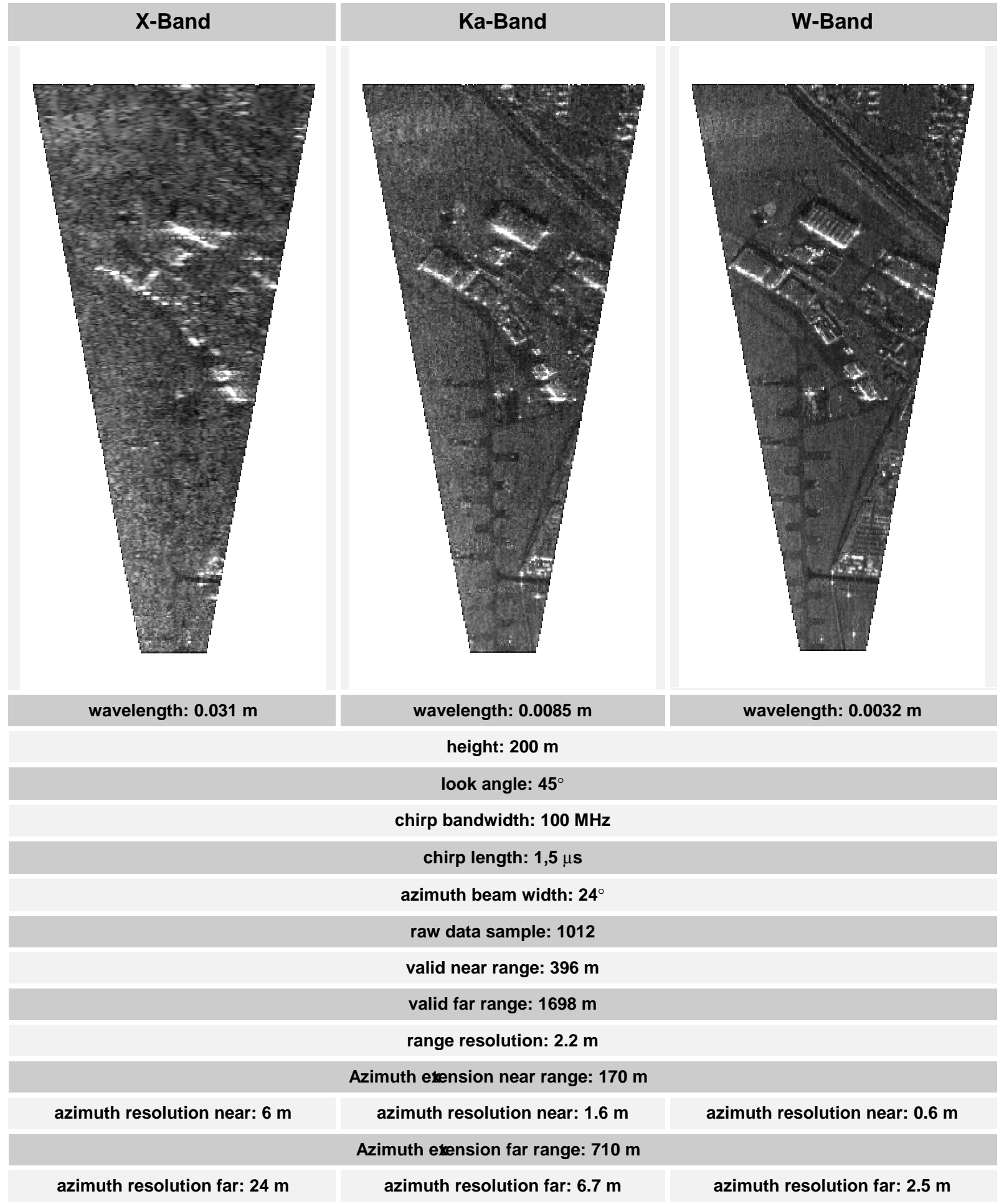

Table 1: SIREV image simulations for X-Band (left), Ka-Band (middle) and W-Band (right). 


\section{Results from an experimental model}

In order to prove the feasibility of the SIREV principle, a functional model has been built up at the German Aerospace Center [1]. To minimize the costs it was preferable to use existing radar hardware even with specifications that were not optimized for this purpose. For example, a sequential switching of the 56 receiving antenna elements was necessary and the experimental model had to be built up in X-Band notwithstanding the poor image resolution to be expected due to the short antenna length of $2.85 \mathrm{~m}$. Details of the radar hardware and the antenna system may be found in [1] and [12]. Figure 4 shows the antenna system and its attachment to the helicopter.
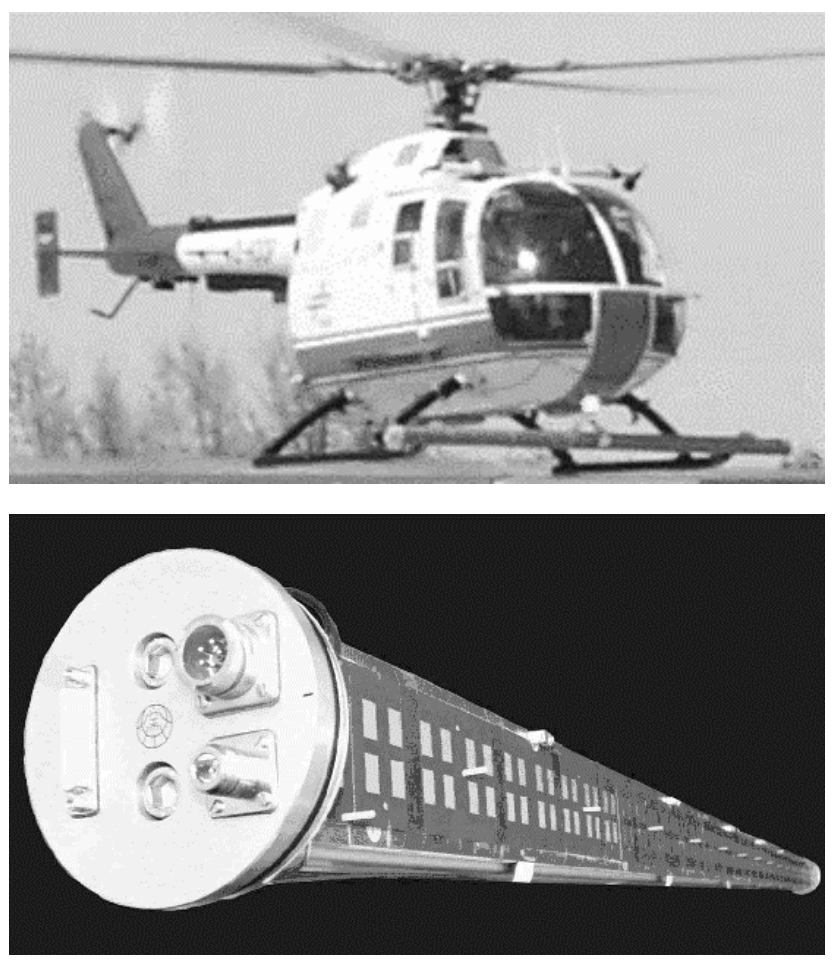

Figure 4: Top: Antenna system attached to the skids of the helicopter. Bottom: RF side of the SIREV antenna with the 5622 patch receiving subarrays. A detailed description of antenna hardware may be found in [12].

In the following, we will report our experimental results from two helicopter flights in the vicinity of Oberpfaffenhofen (Germany). The first scene contained a large corner reflector as the main scattering element and data from this recording were used for a calibration of the system. The second raw data set was obtained during a flight across the river 'Lech'. The recording parameters of these two flights are summarized in Table 2.
Table 2: Recording parameters

\begin{tabular}{|c|c|c|}
\hline & \multicolumn{2}{|c|}{ recorded scene } \\
\hline & $\begin{array}{l}\text { corner } \\
\text { reflector }\end{array}$ & $\begin{array}{l}\text { river } \\
\text { "bch" }\end{array}$ \\
\hline Center frequency & \multicolumn{2}{|c|}{$9.5 \mathrm{GHz}$} \\
\hline Chirp bandwidth & \multicolumn{2}{|c|}{$100 \mathrm{MHz}$} \\
\hline Antenna length & \multicolumn{2}{|c|}{$2.85 \mathrm{~m}$} \\
\hline $\begin{array}{l}\text { Number of receive antenna } \\
\text { elements }\end{array}$ & \multicolumn{2}{|c|}{56 (equally distributed) } \\
\hline PRF (sequential switching) & \multicolumn{2}{|c|}{$14793 \mathrm{~Hz}$} \\
\hline Chirp duration & $1.3 \mu \mathrm{s}$ & $1.3 \mu \mathrm{s}$ \\
\hline Average helicopter velocity & $28 \mathrm{~m} / \mathrm{s}$ & $21 \mathrm{~m} / \mathrm{s}$ \\
\hline Average helicopter altitude & $1056 \mathrm{~m}$ & 927 m \\
\hline Average ground level & $556 \mathrm{~m}$ & $645 \mathrm{~m}$ \\
\hline
\end{tabular}

\subsection{Raw data analysis}

In order to test the integrity of the recorded raw data set we first investigated the response to the large corner reflector. An excerpt of the demodulated complex format raw data is shown in Figure 5 on the left, where the dominant chirp signals from the corner reflector are easily recognized.

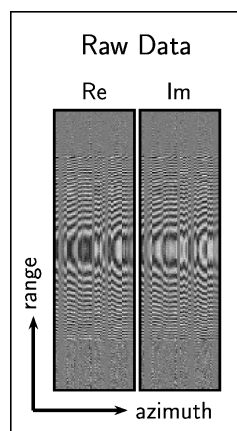

(a)

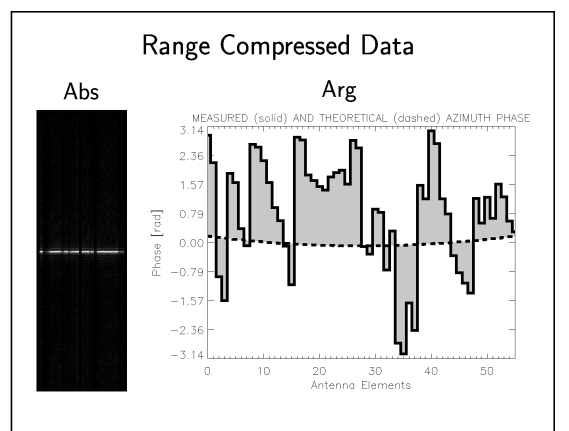

(b)

(c)
Figure 5: Raw data analysis of the corner reflector response. (a) Exerpt of the recorded raw data in complex format. (b) Magnitude of the range compressed data. (c) Comparison of the measured (solid line) and the simulated (dashed line) azimuth phase response curves for a corner reflector located at an azimuth angle of zero degrees and a slant range distance of $891 \mathrm{~m}$.

From a visual inspection of the raw data an important aspect becomes immediately apparent: the recorded azimuth phases suffer from substantial discontinuities, which manifest themselves as disruptions between the individual range lines. These phase errors, which are mainly caused by the different wiring and radiation 
properties of the individual antenna elements, are a major concern, since they will substantially degrade the image quality. To quantify the phase errors, we first compressed the received radar signals in range and then extracted the azimuth phase response of the corner reflector (Figure 5, b and c). In this representation, large phase discontinuities up to $\pm \pi$ become apparent. For comparison, the analytically derived azimuth phase function of an ideal point target at azimuth position $y_{0}=0 \mathrm{~m}$ and slant range $r_{0}=761,5 \mathrm{~m}$ is also plotted in this figure using a dashed curve. Improved focusing will be achieved for all recorded frames by multiplying each recorded range line with a complex phasor, which is immediately derived from the difference of the measured azimuth phase and its theoretical prediction. The successful focusing by this method is demonstrated in Figure 6, where we compare an example of one compressed corner reflector response with the impulse response function of an ideal point target located at $y_{0}=9,6 \mathrm{~m}$ and $r_{0}=681,8 \mathrm{~m}$. The raw data frame was recorded approx. 3 seconds after the frame on which the phase correction is based.
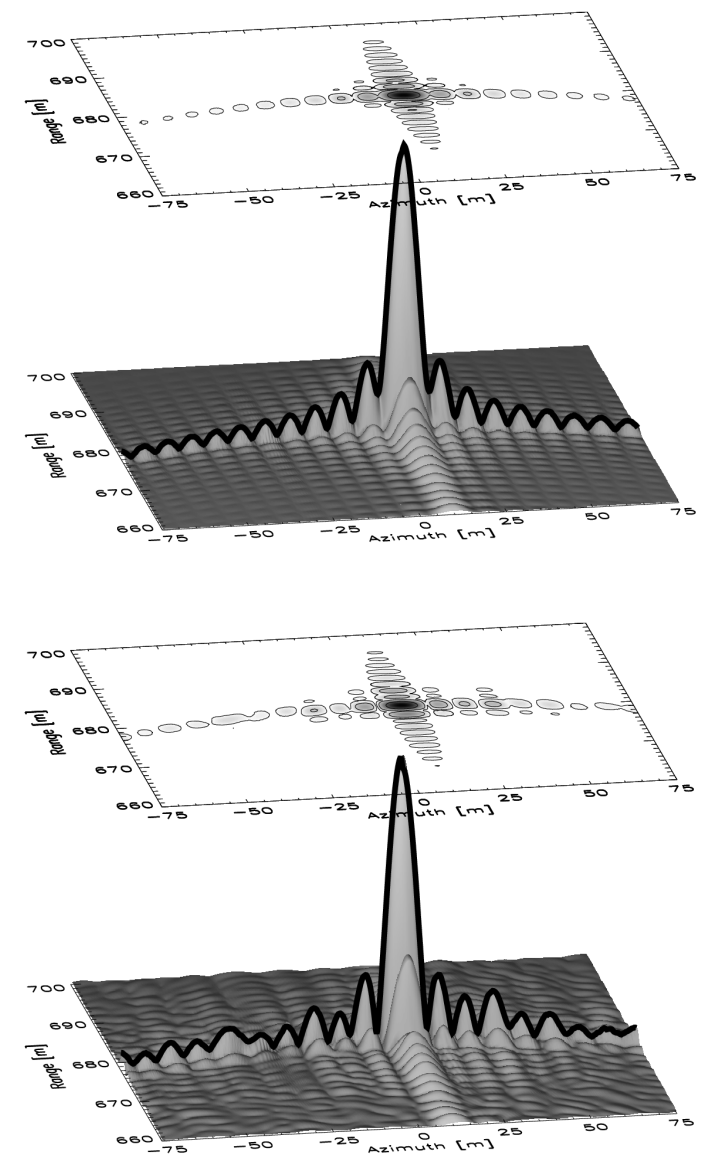

Figure 6: Comparison of the theoretical (top) and measured corner reflector response (bottom).
In the phase compensation algorithm introduced above, we have implicitly assumed that all phase errors are both time- and space-invariant. In order to test this hypothesis we have extracted the compressed corner reflector responses for a sequence of 10.000 frames corresponding to a flight period of 37,9 seconds. A visualization of the azimuth profiles of these corner reflector responses is shown in Figure 7 as a 3-D surface plot.

From the temporal sequence of the azimuth profiles in Figure 7 it becomes apparent, that the focusing is almost independent of both the azimuth angle and the frame number, thus supporting the hypothesis of spaceand time-invariant phase errors. However, a closer look into the corner reflector responses revealed some small but systematic space-variant errors, which are presumably due to variations in the phase pattern of the receiving array antenna. In principle, a compensation of these space-variant azimuth phase errors may be incorporated in the correlation algorithms introduced in Section 3.1, where we apply for each pixel in the output image a different reference function. Since an accurate implementation of this technique requires knowledge of a large number of phase response curves for a densely sampled grid of azimuth and elevation angles, we have restricted the phase error correction to the space-invariant phase shift of the range lines as described before.

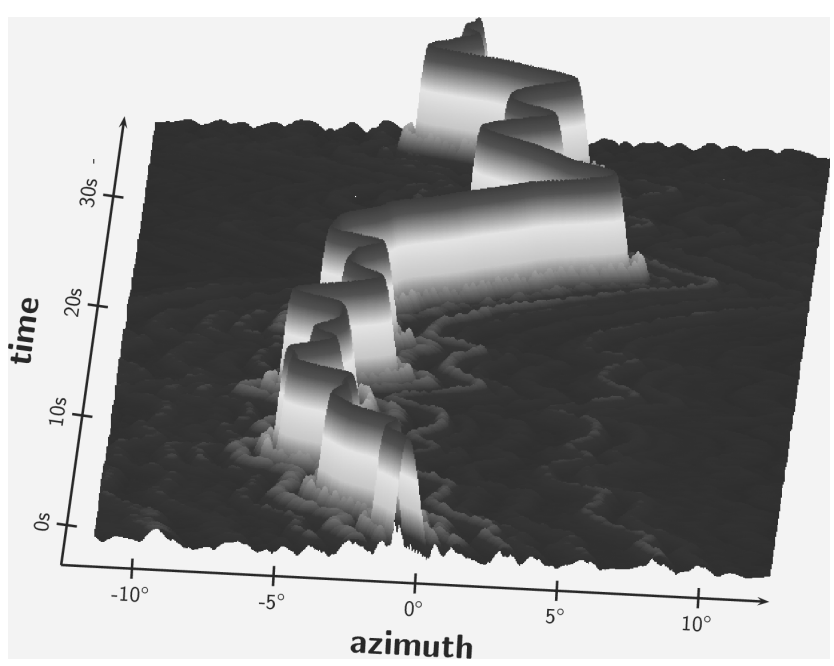

Figure 7: Temporal sequence of corner reflector responses obtained as the helicopter moved froward. The azimuth profile of the focused radar image is shown as a function of azimuth angle and frame number. 


\subsection{Image processing results}

In the preceding subsection we have demonstrated that a successful focusing of the recorded SIREV raw data is achieved, if the phase disruptions between the individual range lines are appropriately compensated. Using the phase corrections derived from the corner reflector response, we have also processed the raw data of the flight across the river 'Lech'. An example of an image obtained by the SIREV processing is shown in Figure 8(a).

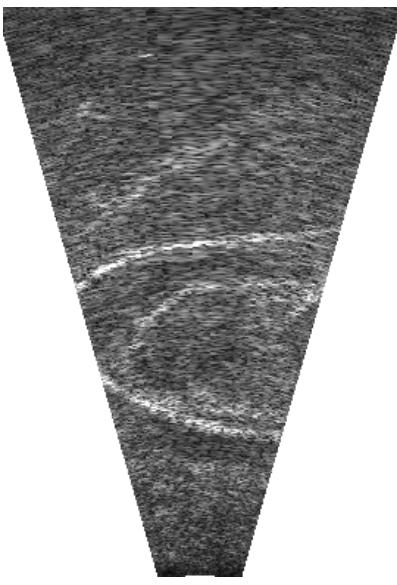

(a)

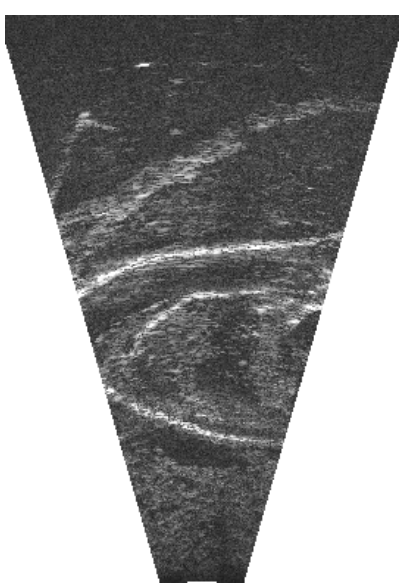

(c)

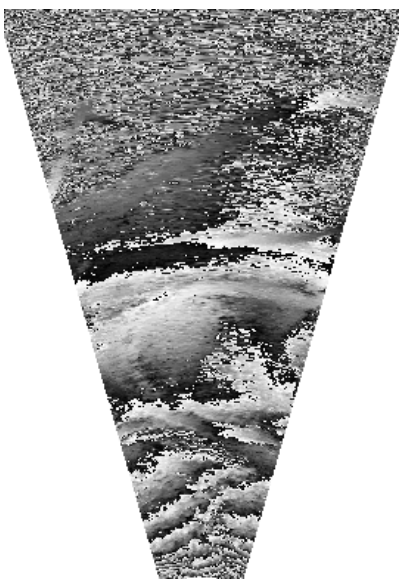

(b)

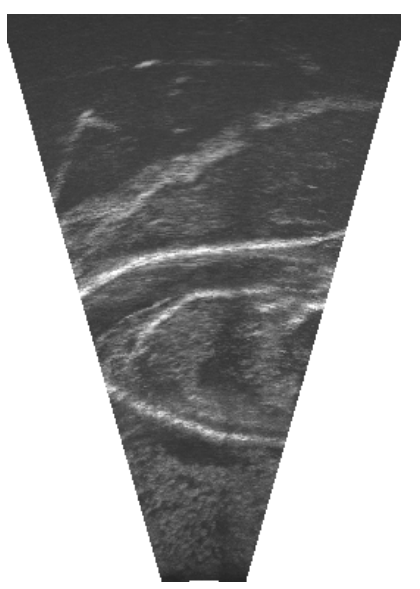

(d)
Figure 8: Results of SIREV processing: (a) Focused image. (b) Average phase offset. (c) Coherent smoothing with $\mathrm{N}=50$. (d) Hybrid smoothing.

The displayed image has an extension of $1150 \mathrm{~m}$ in slant range. The azimuth extensions are $163 \mathrm{~m}$ in near range and $790 \mathrm{~m}$ in far range. From the processed image it becomes apparent, that system noise is a major concern. The low SNR was mainly caused by the sequential switching and the low gain of the receiving antenna elements.

\subsection{Noise suppression}

As we have seen in the preceding section, the processed SIREV images suffer from a substantial contamination with noise. To alleviate these deteriorating effects, we took advantage of the high image repetition frequency $\left(f_{\text {img }}=P R F / 56=264 \mathrm{~Hz}\right)$ by appropriately combining the information of consecutively recorded frames. Most conveniently, this is achieved by a linear summation over several frames. In order to compensate for systematic phase shifts induced by the helicopter movement, an appropriate phase correction has to be applied prior to complex summation. In our algorithm, which will be explained in detail below, this phase correction is automatically extracted from the image sequence. As an alternative, the necessary phase offsets could be deduced from the motion parameters of the helicopter. The block diagram in Figure 9 summarizes the algorithm, which consists of three processing steps.

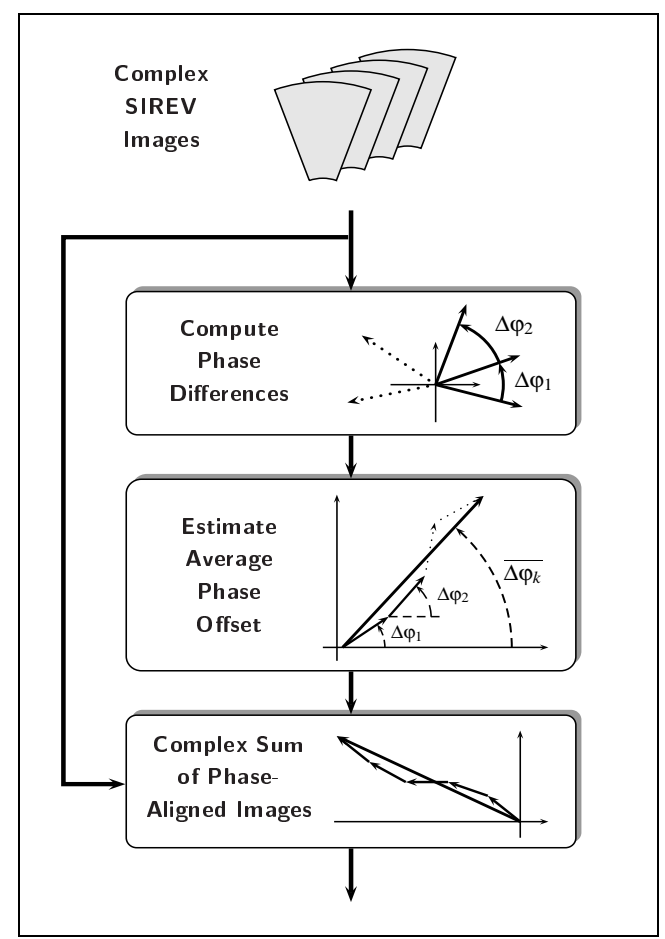

Figure 9: Block diagram of coherent SIREV image smoothing procedure.

The coherent SIREV smoother starts with a set $\mathbf{u}=\left\{u_{1}(y, r), \ldots, u_{N}(y, r)\right\}$ of $N$ complex valued images denoted by $u_{i}(y, r)$. From this set phase differences 
$\Delta \varphi_{i}(y, r)$ between successive image pairs are computed using complex multiplication and taking the argument (phase) of the result:

$$
\Delta \varphi_{i}(y, r)=\arg \left[u_{i}(y, r) \cdot u_{i+1}^{*}(y, r)\right]
$$

This yields a set of $N-1$ phase differences which may be visualized in 2-D space by $N-1$ vectors of unit length. An illustration of this processing step is shown within the first block of Figure 9. Taking advantage of the vector representation of complex numbers, an average phase offset may be computed using 2-D vector summation as illustrated in the second block of Figure 9. Analytically, this can be written as

$$
\overline{\Delta \varphi(y, r)}=\arg \left\{\sum_{i=1}^{N-1} \exp \left[j \Delta \varphi_{i}(y, r)\right]\right\}
$$

where $\overline{\Delta \varphi(y, r)}$ denotes the two-dimensional average phase offset. An example of the spatial phase distribution obtained by this 2-D phase smoothing process is provided in Figure $8(\mathrm{~b})$, where $\overline{\Delta \varphi(y, r)}$ has been computed for a set of $N=50$ complex images.

The average phase offset function $\overline{\Delta \varphi(y, r)}$ is now used to correct the phase of the individual complex images for possible shifts due to movements of both the transmit and the receive antennas. This can be achieved by multiplying each input image $u_{i}(y, r)$ with a complex phase factor $\underline{\varphi}_{i}(y, r)$ which is immediately derived from the average phase offset $\overline{\Delta \varphi(y, r)}$ :

$$
\underline{\varphi}_{i}(y, r)=\exp [-j(i-1) \overline{\Delta \varphi(y, r)}]
$$

In this equation, a linear motion of both the receiving and the transmitting antennas has been assumed, resulting in an approximately linear decrease of the phase values as the helicopter moves forward. In a final step, the $N$ phase aligned complex images are coherently summed according to the formula

$$
\begin{aligned}
\overline{u(y, r)} & =\sum_{i=1}^{N} u_{i}(y, r) \cdot \underline{\varphi}_{i}(y, r)= \\
& =\sum_{i=1}^{N} u_{i}(y, r) \cdot \exp [-j(i-1) \overline{\Delta \varphi(y, r)}]
\end{aligned}
$$

which yields the smoothed image $\overline{u(y, r)}$. An example of the resulting image obtained by this complex averaging process is shown in Figure 8(c), where the number of coherently summed images is $N=50$. It becomes apparent that the coherent smoothing technique substantially improves both the image contrast and the SNR.
The coherent smoothing process described above may also be combined with an incoherent average, as given by a linear summation of several detected images after coherent summation. The result of this hybrid smoothing technique is shown in Figure $8(\mathrm{~d})$ for a total of 50 coherently and 50 incoherently averaged images. As can be seen from the last image in Figure 8 the incoherent summation further reduces the noise at the cost of a slightly impaired image resolution. The deterioration in geometric resolution may be avoided by an appropriate spatially inhomogeneous shift of the individual images which basically compensates for the helicopter movement. This 'co-registration' allows also the number of averaged images to be increased, which will further improve the quality of the resulting image.

\subsection{Display geometries}

The images shown in Figure 8 are represented as a function of azimuth and slant range. More natural from a pilots point of view is a representation in 'optical coordinates' which may be obtained by an appropriate central perspective projection (Figure 10, see also [1]). An example for this geometrical image tranformation is given in Figure 11 together with an optical image of the recorded scene.

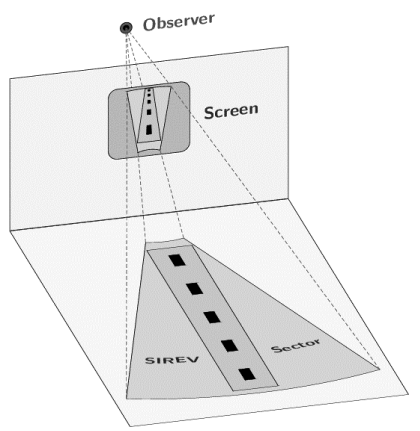

Figure 10: Cyclopean projection from ground coordinates to screen coordinates.
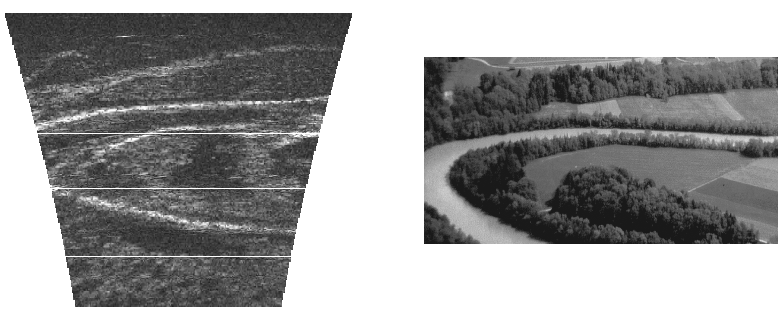

Figure 11: Comparison of the 'cyclopean' SIREV image representation with an optical image. Left: Excerpt of the pilot's view obtained from the SIREV image shown in Figure 8c. Right: Optical image recorded during a separate flight from a slightly different height and viewing angle. 


\section{Discussion}

This paper has given a short overview of the data acquisition, modeling and processing of SIREV raw data. The final result of the experimental model was a movie, composed of several thousands of successive images as in Figure 8(c), which demonstrates the validity and applicability of the SIREV principle. An excerpt of the movie is accessible on the SIREV Internet homepage [4].

SIREV has a great potential for applications where a radar mapping with short to moderate range distances with a forward looking geometry is required. The image formation is based on a digital beam forming on receive which allows a great flexibility for correcting phase and amplitude errors caused by the radar system or platform induced motion errors. By this, the side-lobes of the impulse response function can efficiently be suppressed even in the case of extremely high phase errors. Additionally, a coherent averaging process can be used to improve the signal to noise ratio of the processed images.

The SIREV principle can also be combined with the SAR principle for a very high squinted imaging geometry. Although it is not possible to improve the azimuth resolution in the middle of the imaging sector, the Doppler effect can be exploited to improve the resolution in the forward direction by forming a synthetic aperture orthogonal to the SIREV antenna. In this case, several image frames have to be stored and the Doppler history can be used due to the coherent nature of the data acquisition scheme. This resolution improvement assumes, however, that the platform has a forward movement which is not necessarily the case for an imaging with the SIREV principle. A downward looking geometry can also be considered in the case of a moving platform [3].

Interferometry may also be implemented by adding a second transmit antenna with a baseline in the flight direction plane and perpendicular to the line of sight. The same array of receiving antennas as for two-dimensional imaging can be used so that the additional hardware requirements are minimized. In this case, however, an inertial navigation system as well as a precise positioning system like differential GPS is required for accurate height determination. The feasibility of such a system using longer wavelength radar, e.g. for forest biomass estimation, will be investigated in a future work.

\section{References}

[1] S. Buckreuss, G. Krieger, J. Mittermayer, A. Moreira, R. Scheunemann, T. Sutor, M. Wendler, F. Witte, M. Younis, Y. Venot, W. Wiesbeck, SIREV - Development of a functional model. DLR Forschungsbericht 2000-44, 2000.

[2] J. C. Curlander and R. N. McDonough, Synthetic Aperture Radar: Systems and Signal Processing. New York: Wiley, 1991.

[3] C. H. Gierull, "Combining the idea of a downwardlooking with a forward-looking airborne imaging radar," in Proc. EUSAR 2000, Munich, pp. 377-380, May 2000.

[4] http://www.dlr.de/hr/sirev/home.html

[5] R. Horn, "The DLR airborne SAR project E-SAR," in Proc. IGARSS, pp. 1624-1628, 1996.

[6] J. Mittermayer, A. Moreira and O. Loffeld, "Spotlight SAR data processing using the frequency scaling algorithm,". IEEE Trans. Geosci. Remote Sensing, vol. 37, no. 5, pp. 2198-2214, 1999.

[7] J. Mittermayer, M. Wendler, G. Krieger, A. Moreira, T. Sutor, and S. Buckreuss, "Data Processing of an Innovative Forward Looking SAR System for Enhanced Visison," in Proc. EUSAR 2000, Munich, pp. 733-736, May 2000.

[8] A. Moreira, J. Mittermayer, and R. Scheiber, "Extended Chirp Scaling Algorithm for Air- and Spaceborne SAR Data Processing in Stripmap and ScanSAR Imaging Modes", IEEE Trans. on Geosci. and Remote Sensing, vol. 34, no. 5, 1996.

[9] R. A. Ryerson, Manual of remote sensing. New York: Wiley \& Sons, Inc., 1998.

[10] T. Sutor, F. Witte, and A. Moreira: "A new sector imaging radar for enhanced vision - SIREV," in Proc. SPIE, Vol. 3691, pp. 39-47, 1999.

[11] F. Witte, "Forward looking radar (coherent)," Deutsches Patent Nr. 4007612, 1990, US Patent Nr. 5182562, 1993.

[12] M. Younis and W. Wiesbeck, "Antenna system for a forward looking SAR using digital beamforming onreceive-only," in Proc. IGARSS 00, Honululu, 2000. 